\title{
Growth, osmoregulatory responses and changes to the lipid and fatty acid composition of organs from the mud crab, Scylla serrata, over a broad salinity range
}

\begin{abstract}
Aquatic animals can often undergo substantial physiological responses to salinity; however, associated lipid/fatty acid alterations to their various tissues have received little attention. To investigate this, we measured the growth of mud crab, Scylla serrata, juveniles over two moults (duration of 23-60 days) at salinities of 4, 12, 20, 28, 36 and 44\% (30 replicates/treatment). After the second moult, 6-day post moult crabs were sampled for hepatosomatic index (HSI), haemolymph osmolality, $\mathrm{Na}+\mathrm{K}+$ and $\mathrm{Ca} 2+$ levels, anterior/posterior gill $\mathrm{Na}+\mathrm{K}+$-ATPase activity as well as the lipid/fatty acid composition of the anterior and posterior gills, hepatopancreas and muscle. High salinities of 36 and 44\%o significantly lowered crab growth and HSI $(\mathrm{p}<0.01)$. S. serrata strongly hyper-regulated haemolymph osmolality and ions likely due to significantly enhanced posterior gill $\mathrm{Na}+\mathrm{K}+-$ ATPase activity. At decreasing salinities, eicosapentaenoic acid, arachidonic acid, n-3 and long chain polyunsaturated fatty acid (PUFA) significantly increased $(p<0.05)$, likely to maintain $\mathrm{Na}+\mathrm{K}+-\mathrm{ATPase}$ activity via increased membrane fluidity. Muscle and hepatopancreas n-3/n-6 PUFA ratios significantly decreased $(\mathrm{p}<0.05)$ at increasing salinities indicating possible fatty acid metabolic modifications. Results indicate that S. serrata juveniles are well adapted to low salinities, with higher salinities likely reducing their metabolism, reflected by lower growth, HSI and higher posterior gill and hepatopancreatic triacylglycerol.
\end{abstract}

Keyword: Scylla serrata; Osmoregulation; Salinity; Fatty acid; Na+/K+-ATPase activity 